NASA Technical Memorandum 107060

\title{
A Comparison of Multivariable Control Design Techniques for a Turbofan Engine Control
}

Stephen R. Watts

United Technologies Pratt \& Whitney

West Palm Beach, Florida

and

Sanjay Garg

Lewis Research Center

Cleveland, Ohio

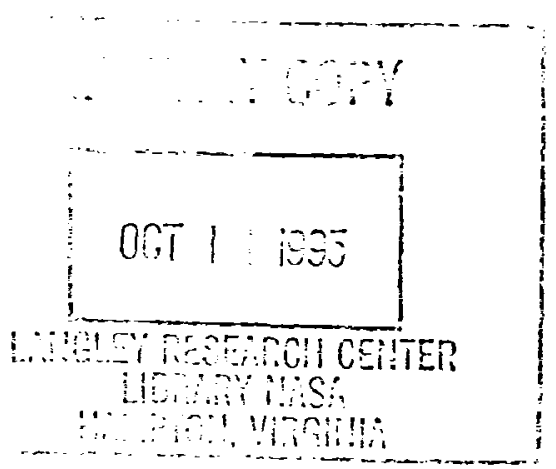

Prepared for the

40th Gas Turbine and Aeroengine Congress and Exposition sponsored by the American Society of Mechanical Engineers

Houston, Texas, June 5-8, 1995

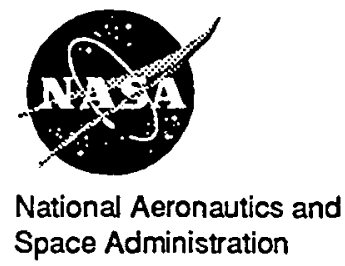




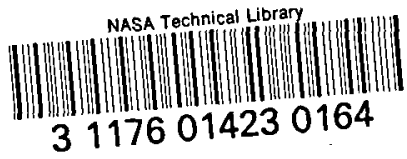

\title{
A COMPARISON OF MULTIVARIABLE CONTROL DESIGN TECHNIQUES FOR A TURBOFAN ENGINE CONTROL
}

\author{
Stephen R. Watts \\ United Technologies Pratt \& Whitney \\ Government Engine and Space Propulsion \\ West Palm Beach, Florida \\ Sanjay Garg \\ Advanced Control Technology Branch \\ NASA Lewis Research Center \\ Cleveland, Ohio
}

\begin{abstract}
This paper compares two previously published design procedures for two different multivariable control design techniques for application to a linear engine model of a jet engine. The two multivariable control design techniques compared were the Linear Quadratic Gaussian with Loop Transfer Recovery (LQG/LTR) and the H-Infinity $(H \infty)$ synthesis. The two control design techniques were used with specific previously published design procedures to synthesize controls which would provide equivalent closed loop frequency response for the primary control loops while assuring adequate loop de-coupling. The resulting controllers were then reduced in order to minimize the programming and data storage requirements for a typical implementation. The reduced order linear controllers designed by each method were combined with the linear model of an advanced turbofan engine and the system performance was evaluated for the continuous linear system. Included in the performance analysis are the resulting frequency and transient responses as well as actuator usage and rate capability for each design method. The controis were also analyzed for robustness with respect to structured uncertainties in the unmodeled system dynamics. The two controls were then compared for performance capability and hardware implementation issues.
\end{abstract}

\section{Introduction}

Future fighter aircraft designs will require highly responsive propulsion systems to achieve superior agility and maneuverability. In order to meet the increased performance requirements, propulsion system manufacturers are migrating away from traditional single input single output control architectures and are beginning to implement and test linear multiple input multiple output multivariable control architectures in their technology programs.

The Linear Quadratic Gaussian with Loop Transfer Recovery (LQG/LTR) and H-Infinity $(H \infty)$ design techniques are well known and well documented in the literature. Numerous references are available for the mathematical formulation and sample applications of these two design techniques with specific application procedures (Athans, 1986 and Garg, 1993). Both of these design techniques have been reviewed when applied to aircraft flight control systems, this paper reviews the design techniques and application procedures when applied to the turbofan engine control problem. The work described berein compares the resulting overall system performance and implementation issues for the two multivariable control designs and is not intended to be a detailed review of the design steps for the two design procedures, nor does it suggest any possible variations to these design procedures. Rather, it highlights the design procedures and provides all pertinent references required to recreate the analysis.

The discussion is organized in the following manner. The linear engine model and actuator model that are used for the controller synthesis will be defined. The selected control mode for the controller synthesis will be identified. The two controller synthesis techniques will be briefly outlined. The method for reducing the controller order will then be discussed, and the computational requirements of the reduced order controllers will be reviewed. These controllers will then be exercised transiently and system performance will be compared. The issue of robustness for structured uncertainties in the unmodeled system dynamics will then be explored. Finally, the two controllers will be compared in terms of overall performance capabilities.

\section{Engine Model}

The engine model used in the control design is linear model of an advanced afterburning urbofan engine at maximum non-augmented power. The engine model is represented in the following state space form:

$$
X=A \operatorname{eng} X+B \operatorname{eng} U
$$




$$
Y=C_{e n g} X+D_{e n g} U
$$

where the state vector is

$$
X=[N 1, N 2, T M H P T]^{T}
$$

and the states are defined as:

N1 = Low Pressure Compressor Speed (RPM)

N2 = High Pressure Compressor Speed (RPM)

TMHPT = Metal Temperature of the High Pressure Turbine (Degrees C).

The control input vector is

$$
\mathrm{U}=[\mathrm{WF}, \mathrm{AJ}, \mathrm{CIVV}, \mathrm{RCVV}]^{\mathrm{T}}
$$

where the inputs are defined as:

$$
\begin{aligned}
& \text { WF = Main Bumer Fuel Flow (Kg/HR) } \\
& A J=\text { Nozzle Exit Area }\left(M^{2}\right) \\
& C I V V=\text { Low Compressor Inlet Variable Guide } \\
& \text { Vanes (Degrees) } \\
& \text { RCVV = Rear Compressor Inlet Variable Guide } \\
& \text { Vanes (Degrees). }
\end{aligned}
$$

The output vector is :

$$
\mathrm{Y}=[\mathrm{OPR}, \mathrm{EPR}, \mathrm{N} 1, \mathrm{~N} 2]^{\mathrm{T}}
$$

and the outputs are defined as:

OPR = Ratio of Burner Pressure/lnlet Pressure (Dimensionless)

$\mathrm{EPR}=$ Ratio of Nozzle Pressure /Inlet Pressure (Dimensionless)

N1 = Low Pressure Compressor Speed (RPM)

N2 = High Pressure Compressor Speed (RPM).

OPR, EPR and N2 are the sensed outputs for the control loops and $\mathrm{N} 1$ is used for the inner loop scheduling for the Low Compressor Inlet Variable Vanes (CIVV).This model is a perturbation model and model inputs and outputs are deltas from the nominal operating point. CIVV is scheduled open loop as a function of NI and is included in the synthesis because of its' transient effects on EPR. The scale factor for CIVV/N1 is 0.01244 Degrees/RPM. The numerical values for the system matrices: Aeng. Beng, Ceng, Deng, for the engine model are listed in the appendix.

\section{Actuator Model}

The actuator dynamics are represented as first order lags with loop gains of 25 radians/second for the WF, CIVV, and RCVV loops. The $A J$ actuator loop is represented by a second order system with $\omega=0.45$ and $\zeta=55.8$ in series with a first order lag with a loop gain of $15 \mathrm{radians} / \mathrm{second}$. See Figure 1 for the schematic view of the integrated engine /actuation system.

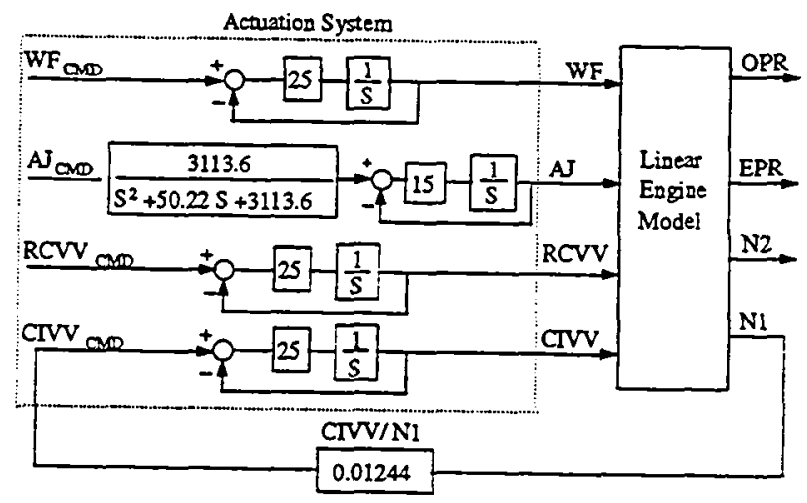

\section{FIGURE 1. INTEGRATED ENGINE/ACTUATION SYSTEM OVERVIEW}

\section{Control Mode}

The engine transient control mode was selected because of inherent properties for directly controlling the engine operating line during transient operation (Larkin, 1994), while providing rapid precise control of engine thrust. The three control loops are the ratio of burner pressure to inlet pressure (OPR), the ratio of nozzle pressure to inlet pressure (EPR), and High Rotor Speed (N2). The Controller outputs are main bumer fuel flow (WF), nozzle exit area (AJ) and rear compressor inlet variable guide vanes (RCVV). The control specification is to track the input commands while maintaining zero steady-state error in a de-coupled manner. The desired bandwidths for all the control loops are 10 (radians/second).

\section{Controller Synthesis}

These two controller synthesis procedures are of particular interest because of the ease in solving multivariable control problems with available commercial software analysis tools to assist in the numerical computations. These procedures require a minimal amount of effort to setup the design plants and the computations can easily be repeated for numerous applications without significant changes to the design setup. Both LQG/LTR and $H \infty$ controller synthesis procedures were completed using MATRIXx with Robust Control Module, and other in house analysis tools.

The LQG/LTR synthesis was completed using the method described in (Athans, 1986). In this method, the calculation of the LQG/LTR controller is straight forward in that the control system designer specifies a properly scaled nominal plant model and appends the necessary integrators to meet the command following and disturbance rejection performance specifications. The control system designer defines a target feedback which recovers the controller outputs loop via a Kalman Filter and solves the Ricatti equation to obtain the full state feedback gain matrix. See Figure 2 for the LQG/ 
LTR compensator structure. In Figure 2, A, B and C represent the matrices for the design plant. Additionally, this LQG/LTR controller synthesis provides some highly desirable stability guarantees, 60 degrees phase margin and $6 \mathrm{~dB}$ gain margin. This particular synthesis does not however directly address any control usage and control rate considerations. The concept of control usage and control rates will be discussed further in the Performance Analysis Section.

The LQG/LTR design requires a properly scaled nominal plant. Garg, (1989) discusses the importance of scaling the nominal plant in terms of the singular values of the controller outputs. Improperly scaled nominal plants can result in very poor target feedback loops and thus poor controllers. Other design techniques (Larkin, 1985) which recover the target feedback loops at the controller inputs do not require the same attention to scaling to obtain good target feedback loops. The scale factors were chosen as the inverse of the nominal operating point for the engine model inputs and outputs, except for the RCVV scale factor. The RCVV range of motion is -35.0 to +5.0 degrees, and the initial condition of the control input for the model selection is 0.0 . Therefore the inverse of the range of motion was chosen as the scale factor for the RCVV loop. The complete actuator dynamics were included in the LQG/LTR nominal plant model.

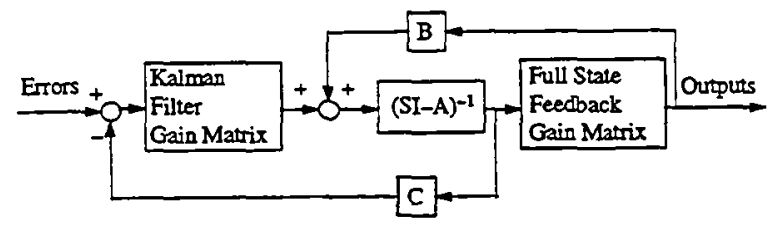

\section{FIGURE 2. LQG/LTR COMPENSATOR STRUCTURE}

This particular LQG/LTR synthesis does not allow for non-zero D Matrix in the nominal plant. Therefore, two first order lags with 1 millisecond delays were added to the OPR, and EPR outputs to remove any direct feed through terms in the systems outputs (a zero D Matrix). Also the properly scaled plant was augmented with three integrators. Integrators were added in each of the control loops to provide for command tracking and zero steady-state error to step commands. The complete LQG/LTR augmented scaled design plant is shown in Figure 3. In Figure 3, Su and Sy are the scale factors for the inputs and the outputs respectively. The numerical values for Su and Sy are listed in the appendix.

Obtaining an acceptable controller required a minimal number of iterations. After controller synthesis was completed the bandwidth specification was checked, the target feedback loop design was modified and the process was repeated until design specifications were met. The LQG/LTR synthesis resulted in a controller which met the 10 radian/second bandwidth specification while maintaining loop de-coupling. This particular design procedure requires the inclusion of integrators in the scaled design plant and then the inclusion of another set of integrators in the controller implementation. The resulting LQG/LTR controller was 17 th order, the three states of engine model, six states of the acturator model and three additional states for the augmented plant and three states for controller integrators to account for the augmented plant.

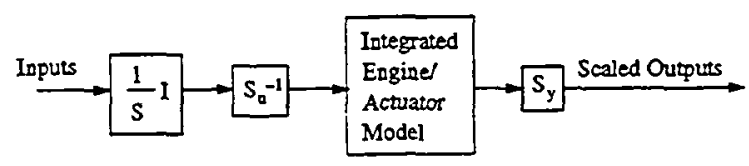

\section{FIGURE 3. AUGMENTED SCALED LQGLTR DESIGN PLANT}

The $H \infty$ engine control design problem is formulated as a command tracking, disturbance rejection problem within the framework of the general mixed sensitivity $H$ o control problem (Chiang and Satonov, 1988). The detailed block diagram for the $H \infty$ formulation of the engine control design is shown in Figure 4. In Figure 4, the controller variables are $Z$ and the corresponding reference commands are $Z_{c}$. The three transfer functions that are of interest for such a problem are the sensitivity function $S(s)$, the complimentary sensitivity function $T(s)$, and the control transmission function $C(s)$. These represent the closed loop transfer functions from the reference commands and disturbances to the tracking errors, controlled variables and commanded control inputs, respectively. In order to influence both the low frequency and high frequency properties of the closed loop system, it is desirable to obtain a controller $\mathrm{K}(\mathrm{s})$ which minimizes a weighted norm of the combination of these three transfer functions, i.e.

$$
\begin{aligned}
& \underset{\text { stabilizing } \mathrm{K}(\mathrm{s})}{\min (\mathrm{s}) \|_{\infty}} \\
& \text { with } \mathrm{H}(\mathrm{s})=\left[\begin{array}{ll}
W_{S}(s) \cdot S(s) \\
W_{T}(s) \cdot T(s) \\
W_{C}(s) \cdot C(s)
\end{array}\right]
\end{aligned}
$$

where $\|\mathrm{H}(\mathrm{s})\|_{\infty}=\sup _{\omega}\left(\sigma_{\max }[H(j \omega)]\right)$.

The terms $\mathrm{W}_{\mathrm{S}}(\mathrm{s}), \mathrm{W}_{\mathrm{T}}(\mathrm{s})$, and $\mathrm{W}_{\mathrm{C}}(\mathrm{s})$ are the weighting functions that the control designer uses as "knobs" to tune the controller $\mathrm{K}(\mathrm{s})$ such that the control design objectives are met. For the command tracking and disturbance rejection problem at hand, $\mathrm{W}_{\mathrm{S}}(\mathrm{s})$ should be chosen to be large at low frequencies and small at high frequencies to ensure good command tracking, while $W_{\mathrm{T}}(\mathrm{s})$ should be chosen small at low frequencies and large at high frequencies to ensure robustness to high frequency unmodeled dynamics, and $W_{C}(s)$ is 
chosen to ensure that achievable actuation bandwidths and control rates and control usage are obtained in the controller $K(s)$.

The $H \infty$ synthesis requires normalization of the plant inputs and outputs, so that the controller calculation is based upon the unity inputs and outputs in the norm that is being minimized. The normalization factors are chosen by a simple analysis of the engine model that requires stepping each of the engine model inputs (WF, AJ, RCVV) either ten percent of their base values or ten percent of the full range of motion and observing the change in the engine model outputs which are used as controller inputs (i.e., OPR, EPR, N2). The inverse of the maximum of the changes in the controller inputs is then selected as the output normalization factors, and the maximum of the changes in the controller inputs is selected as the command normalization factor. The control usage and control rate weighting factors form the control transmission weighting factors $W_{C}(s)$. The control usage weighting factors are the inverse of either ten percent of the initial condition or ten percent of the full range of motion, as discussed previously. The control rate weighting factors are derived as the inverse of the actuator rate capability. The sensitivity and complimentary sensitivity weighting factors, $W_{S}(s)$ and $\mathrm{W}_{\mathrm{T}}(\mathrm{s})$ are used to "dial in" the control loop bandwidths. A complete listing for the $H \infty$ design plant weighting factors is included in the appendix.

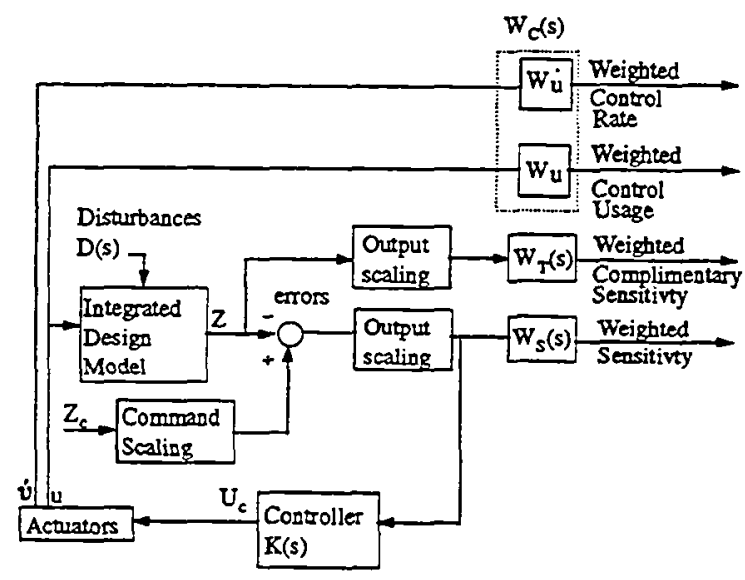

FIGURE 4. Ho DESIGN PLANT

The $H \infty$ synthesis was completed using the method described in (Garg, 1993). Obtaining a suitable controller required a minimal number of iterations. After the controller synthesis was completed the bandwidth specification was checked. The sensitivity and $\infty 0 m-$ plimentary sensitivity weighting factors, $W_{S}(s)$ and $W_{T}(s)$, were adjusted and the process was repeated. The $H \infty$ synthesis resulted in a controller which met the $10 \mathrm{radian} / \mathrm{second}$ bandwidth specification, while maintaining loop de-coupling. The sole objective of the synthesis was to provide a controller which provided the specified bandwidth. Therefore, it should be noted that the control usage and rate weighting factors were not altered during the controller tuning process. The resulting $H \infty$ controller was 15 th order, three states for the engine, six states for the actuator model, three states for the sensitivity weighting functions, and three states for the complimentary sensitivity functions.

A sample of the closed loop frequency response of the OPR loop for both the $H \infty$ and LQG/LTR controllers is shown in Figure 5. Figure 5 shows that the both controllers have approximately 10 radians/second closed loop frequency response, with adequate loop de-coupling. One significant item which should be noted is the relative rate of "roll off" for the command loops. The $H \infty$ shows a faster roll off rate than the LQG/LTR. The LQG/LTR design procedure defines target feedback loops which provide a $20 \mathrm{~dB} / \mathrm{dec}$ ade roll off rate which is then recovered in the LQG/LTR controller loops. The $H \infty$ controller design procedure provides a roll off rate which can be adjusted through the combination of the sensitivity and complimentary sensitivity weighting factor functions which can be adjusted as necessary by the designer.

\section{Controller Reduction}

The $H \infty$ and LQG/LTR controller are both relatively large, 15th and 17th order respectively. Real time implementation issues such as data storage and processor through put capability require that the controller size be minimized. Several reduction techniques are available for minimizing the controller size. Modal residualization, internally-balanced reduction and a frequency weighted intemally balanced reduction technique (Enns, 1984) were reviewed for each application.

The LQG/LTR controller reduction required several attempts. A modal residualization resulted in an $111 \mathrm{~h}$ order controller. The frequency weighted internally balanced reduction technique provided no additional reduction. It should be noted that the inclusion of all the actuator dynamics could be modified to only include the AJ dynamics, as these dynamic effects present significant dynamic interactions at 15 radians/second. But this would result in a full order controller that is 15th order in size and could only be reduced to an 9th order controller. It was expected that either controller could be reduced to less than a 6 th order controller, representative of a second order controller for each of the three control loops. The eigenvalues for the LQG/TR full order controller and the reduced order controller are shown in Table 1.

\section{TABLE 1. LQGRTR FULL ORDER AND REDUCED OR- DER CONTROLLER EIGENVALUES}

\begin{tabular}{|l|l|}
\hline \multicolumn{1}{|c|}{ Full Order } & \multicolumn{1}{|c|}{ Reduced Order } \\
\hline-0.001 & -0.001 \\
\hline-0.001 & -0.001 \\
\hline-0.001 & -0.001 \\
\hline-0.3898 & -0.3898 \\
\hline-2.0596 & -2.0596 \\
\hline-24.594 & -24.594 \\
\hline$-49.042+/-161.95 \mathrm{~J}$ & $-49.042+/-161.95 \mathrm{~J}$ \\
\hline$-137.41+/-114.29 \mathrm{~J}$ & $-137.41+/-114.29 \mathrm{~J}$ \\
\hline-180.63 & -180.63 \\
\hline$-372.67+/-642.15 \mathrm{~J}$ & \\
\hline-755.71 & \\
\hline$-528.88+/-918.39 \mathrm{~J}$ & \\
\hline-1066.2 & \\
\hline
\end{tabular}


The $H \infty$ controller reduction also required several attempts, and it yielded an 8 th order controller. The $H \infty$ controller was firstmodally residualized from a 15 th order to 12 th order. Then a frequency weighted internally balanced reduction produced the final 8 th order controller. Both controller reductions failed to achieve the expected 6 th order or less controller. The eigenvalues for the $H \infty$ full order controller and the reduced order controller are shown in Table 2.

\section{TABLE 2. Ho FULL ORDER AND REDUCED ORDER CONTROLLER EIGENVALUES}

\begin{tabular}{|l|l|}
\hline \multicolumn{1}{|c|}{ Full Order } & \multicolumn{1}{|c|}{ Reduced Order } \\
\hline-0.013930 & -0.013930 \\
\hline-0.014925 & -0.014925 \\
\hline-0.027860 & -0.027860 \\
\hline-0.033598 & -0.3914 \\
\hline-0.39132 & -2.1166 \\
\hline-2.1167 & -25.570 \\
\hline$-2.5197+/-0.3853 \mathrm{~J}$ & $24.555+/-11.226 \mathrm{~J}$ \\
\hline$-24.560+1-11.225 \mathrm{~J}$ & \\
\hline-28.728 & \\
\hline$-39.035+1-61.347 \mathrm{~J}$ & \\
\hline-1779.7 & \\
\hline-2955.5 & \\
\hline
\end{tabular}

The importance of the controller reduction directly effects the application computer in terms of data storage requirements and computer through put capability. Implementation of the $H \infty 8$ th order controller will require data storage for 121 separate gains and implementation of the LQG/LTR 11th order controller will require data storage for 198 separate gains. In a like manner, the computational requirements for the LQG/LTR controller are much larger than that of the $H \infty$ controller. The LQG/LTR will require 1820 computations for each calculation cycle while the $H \infty$ will require 803 computations for each calculation cycle. This assumes that the controller has a non-zero D matrix and no attempts to minimize the controller form, such as converting to the observer canonical format for the controllers was attempted.

The relative data storage requirements and computational requirements clearly indicate the $H \infty$ based controller as the controller of preference if the decision were based upon application issues alone with all other factors being equal. The increasing capability of available computer systems in terms of data storage capability and processor cycle time could very well make this a moot point. $\mathrm{Ob}$ viously, several other issues such as performance and robustness must be reviewed prior to selecting the controller design.

\section{Performance Analysis}

In order to compare the relative merits of two controls with the same closed loop bandwidths, the controls were integrated with the engine model and some small steps were performed for each of the control loops. The objective of the performance analysis is to provide a relative comparison of each controller while performing the same task. Because the two controllers have approximately the same bandwidth specifications, the rise time, transient overshoot and settling time will be directly compared. Additionally, the con- trol usage and rates utilized during these tasks will be directly compared.

The magnitude of the control loop steps was the same as the command scaling used in the $H \infty$ controller synthesis. The step magnitude is a very important consideration in this analysis. The range of Overall Pressure Ratio (OPR) is 1-30.0, the range for Engine Pressure Ratio (EPR) is 1-3.5 and the range for High Rotor Speed (N2) is $0-14000.0 \mathrm{rpm}$. Obviousiy a High Rotor Speed command step change of $1 \mathrm{mpm}$ for $\mathrm{N} 2$ has very little impact on the system, while a step command of 1 unit of EPR has a very large impact, and is unrealistic for the linear model being used. Utilizing the appropriate magnitudes for the step command provides meaningful data for analysis. The magnitudes of the steps were : OPR - 1.6183 (dimensionless), EPR - 0.2553 (dimensionless), N2 - 370.4 (RPM).

The analysis was performed with all the commanded inputs scaled to the specified magnitude and the outputs normalized to the inverse of that same value. Hence, a unit command input will result in a unit output with the normalized system. This will result in normalized control loop parameters while retaining physical engineering units for the controller outputs (WF, AJ, RCVV).

Each control loop was stepped and analyzed independently. The OPR loop step is shown as an example in Figures 6-8. Analysis of each of the control loop step commands indicates the following. The LQG/LTR controller has a slightly faster rise time, 0.05 seconds faster see Figures 6, The LQG/LTR controller also had a slight overshoot in the OPR response (Figure 6).

Both the LQG/LTR and $H \infty$ controllers showed very small perturbations in the inactive controller loops while exercising the command steps (See Figure 6). These perturbations were all less than five percent of the nominal operating point and neither controller showed a distinctive capability over the other in terms loop de-coupling. This verifies the loop de-coupling characteristic as specified in the controller requirements definition.

Figure 7 shows the steady state control usage for each of the steps is similar indicating that the operating point was achieved in the same manner, which is expected for the three input by three output controller. The controller transitioned from the initial condition to the exact same operating condition following the transient.

The control rate activity for the transients was very different. The LQG/LTR routinely required "excessive" control rates to achieve the commanded step response. This excessive control rate activity was not isolated to a single loop. This is illustrated in the commanded step change in the OPR loop (Figure 8), in which the gas generator fuel flow rate, exhaust nozzle area rate and the rear compressor variable inlet guide vane rate for the LQG/LTR controller dwarf the same rates for the $H \infty$ controller. The nominal control rate limit for the gas generator fuel flow loop is $13,636 \mathrm{Kg} / \mathrm{hour}$. The LQG/LTR controller required a gas generator fuel flow rate capability of $31,818 \mathrm{Kg} /$ hour, while the $H \infty$ controller only required a rate capability of $5000 \mathrm{lbm} / \mathrm{hour}$. The nominal exhaust nozzle area control rate limit is $0.51 \mathrm{M}^{2} /$ second. The LQG/LTR controller required an exhaust nozzle area rate capability of $0.48 \mathrm{M}^{2} /$ second while the $H \infty$ controller required a rate capability of $0.04 \mathrm{M}^{2} / \mathrm{second}$. Likewise, the rear compressor variable vane rate limit is 85 degrees/second. The LQG/LIR controller required a rear compressor variable vane 
rate capability of 100 degrees/second while the $H \infty$ controller required a rate capability of 20 degrees/second.

Review of the transient responses for all three steps commands would indicate the transient actuator rate capability requirements for each controller synthesis. The transient actuator rate requirements for implementing these controller can be interpreted as the maximum of the actuator rates from the three steps command response. These maximums along the nominal rates are listed in Table 3. A comparison of the design rates and the required rates for the two controllers for each actuator loop shows, that new design rates would be required or a penalty on the bandwidth specification would result if the LQG/LTR controller were implemented as a result of rate limited operation.

\section{TABLE 3. COMPARISON OF TRANSIENT RATE} REQUIREMENTS

\begin{tabular}{|l|l|l|l|}
\hline $\begin{array}{l}\text { Actuator } \\
\text { Loop }\end{array}$ & $\begin{array}{l}\text { Design } \\
\text { Rate } \\
\text { Capability }\end{array}$ & $\begin{array}{l}\text { LQG/LTR } \\
\text { Rate } \\
\text { Requirement }\end{array}$ & $\begin{array}{l}\text { Ho } \\
\text { Rate } \\
\text { Requirement }\end{array}$ \\
\hline WFGG & 13,636 & 31,818 & 2292 \\
\hline AJ & 0.51 & 0.48 & 0.04 \\
\hline RCVV & 85.0 & 420.0 & 75.0 \\
\hline
\end{tabular}

\section{Robustness Analysis}

The two controls being examined were also analyzed for robustness with respect to the design plant, sensor and actuator uncertainties. The analysis tools, MATRIXX Robust Control Module, used for the robustness analysis require the designer to specify the uncertainties as a function of frequency, $\delta i$, and define the magnitude bounds each of the uncertainties, $l i$, for the uncertain transfer functions to be analyzed. This allows for a cumulative estimation of the overall stability, as a function of frequency, for the closed loop system for all the uncertain transfer functions. The stability margin is then defined as the smallest such that the system can have a pole at $j$, with the uncertain transfer function satisfying

$$
\begin{aligned}
\left(\left(\left|\delta_{i}(j \omega)\right| \leq\right.\right. & \left.\left.\alpha_{i}(\omega)\right)\right): \\
\operatorname{margin}(\omega)= & \max \{\mid \text { system can hole pole at } j \text { with } \\
& \text { magnitude bounds } \left.\alpha l_{i}(\omega)\right\} \\
& \text { (Integrated Systems, Inc., 1991) }
\end{aligned}
$$

This resultant estimation of the overall stability is provided in decibels (dB) and is not an absolute value of stability. Rather, it provides an indication of possible variation in the transfer function uncertainties required to stabilize or destabilize the system. A stability estimation that is less than zero for certain frequencies indicates that some of the uncertain transfer functions will destabilize the system.

The sensor and actuator uncertainties are normally specified in the sensor and actuator design specification requirements. The nominal value for sensor uncertainties was specified as five percent of the sensors input for all frequencies. The nominal value for the actuator uncertainty was specified as 10 percent uniformly for all frequencies. The design plant uncertainties are inherent in the design process used for estimating the linear model of a nonlinear system. For the purposes of this analysis, the design plant uncertainties were defined as the uncertainty between the partial derivatives of the rotor speed rates, with respect to the rotors speed states, and the fuel flow input. An illustration of these uncertainties would be attaching an uncertainty to the partial derivative of $\mathrm{N} 1$ rate, with respect with respect to $N 2$, and attaching an uncertainty to the partial derivative of $\mathrm{N} 1$ with respect to WFGG. This results in six uncertainties being introduced to the design plant.

The result of the stability margin calculation is shown in Figure 9. Both the LQG/LTR and the $H \infty$ controllers are robustly stable for the closed loop system with the uncertainties in the design plant, sensor and actuators. It further indicates that the $H \infty$ controller has an increase in stability margin above the 10 radian/second while the LQG/LTR stability margin maintains almost constant. However, the absolute level of stability margin of both controllers indicates that neither is in any danger of becoming unstable at any frequency for the modeled uncertainties.

\section{Conclusions}

The $H \infty$ and LQG/LTR controllers obtained from the specified design procedures achieved the required bandwidth specification and exhibited similar transient performance in terms of rise time, over/under shoot and settling time. Both controllers also exhibited approximately the same levels of stability margin. The controllers did however vary greatly in how these results were achieved. As shown the LQG/LTR controller required more data storage capability and computations, than the $H \infty$ controller did, to provide the equivalent performance. The LQG/LTR controller required very large transient actuator rate requirements. This resulted in very large positive rate limits followed by large negative rate limits. These LQG/LTR controller rate responses appear as overactive controller rate usage when compared to the $H \infty$ controller. Life cycle cost analysis of these types of actuation systems indicates that unnecessary cycles and large rate capability requirements for an actuation system can result in increased cost and weight and reduced life expectancy, which will necessitate costly, frequent replacement of these systems. The $H \infty$ controller outputs transition smoothly from one operating point to next without overshooting the point, thus not requiring large corrections in the controller outputs.

The $H \infty$ controller synthesis includes control usage and rate weighting factor specifications along with design plant, sensor and actuator uncertainty in the controller synthesis. The LQG/LTR synthesis used for this application does not include any capability for including control rate and usage weighting factors, nor does it include the capability to provide for design plant, sensor and actuator uncertainty. Other LQG/LTR formulations may be available which allow for the inclusion of these factors, but the penalty for this is in the ease of the controller formulation and design plant setup. Thus for the application examined bere, the $H$ co controller synthesis provides superior performance while assuring robustness and minimizing the computational requirements of the controller when compared to this particular LQG/LTR synthesis.

\section{Acknowledgments}

This work was completed while the first author was working an on-site assignment at NASA Lewis Research Center in support of the "Large Engine Technology" Contract, NAS3 26618. The sup- 
port and encouragement provided by Walter C. Merrill of NASA and Ralph Cory of Pratt \& Whitney is gratefully appreciated.

\section{References}

Athans, M., 1986 "A Tutorial on the LQG/LTR Method", LIDS-P-1542, Massachusetts Institute of Technology, Cambridge, MA.

Chiang, R.Y.and M.G. Safonov, 1988 "Robust Control ToolboxFor Use With MATLAB - Users Guide" The Matbworks, INC., Natick, MA.

Enns, D.F., 1984 "Model Reduction for Control System Design", Ph.D. Thesis, Department of Aeronautics and Astronautics, Stanford University, Stanford, CA.

Garg, S., 1993 "Robust Integrated Flight/Propulsion Control for a STOVL Aircraft Using $H \infty$ Control Design Techniques", Auromatica, Vol. 29, No. 1, pp 129-145,

Garg, S., 1989 "Turbofan Engine Control System Design Using the LQG/LTR Methodology", American Controls Conference Proceedings, Vol. 1 pp 134-141.

Integrated Systems, Inc., 1991 "MATRXXX Robust Control Module, edition 2", Santa Clara, CA.

Larkin, L.J., 1985 "Design of a Robust Multivariable Jet Engine Control System", Masters Thesis, College of Engineering, Florida Atlantic University, Boca Raton, Florida

Larkin, LJ., 1994 "Pressure Based Closed Loop Thrust Control in a Turbofan Engine", Patent Number 5,303,545

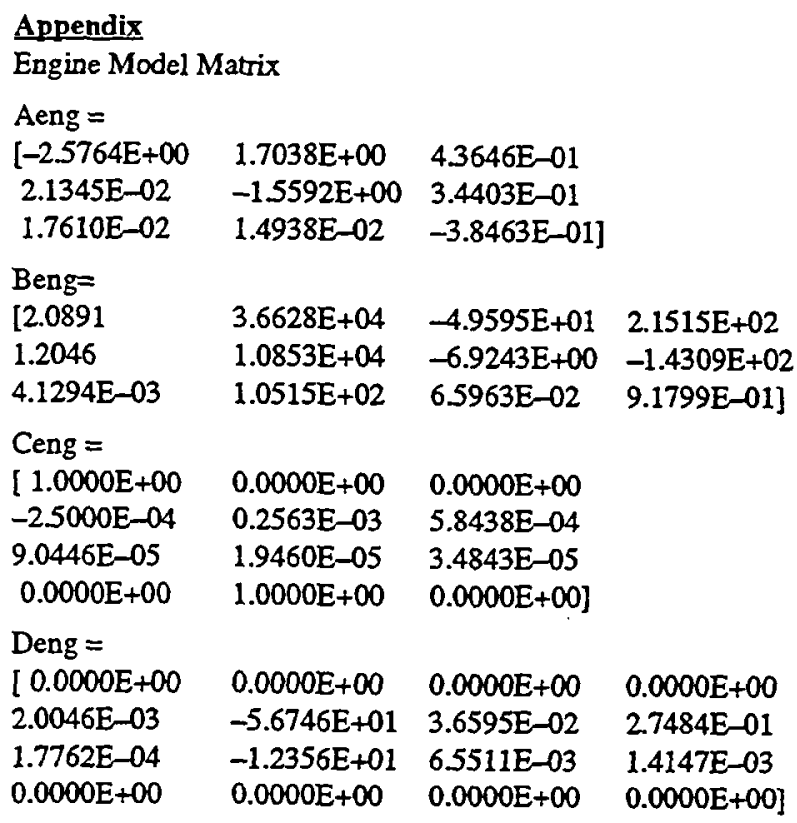

LQGRTR Design Plant Scale Factors

\begin{tabular}{|c|c|c|}
\hline $\mathrm{Su}=[2.6000 \mathrm{E}-0.4$ & $0.0000 \mathrm{E}+00$ & $0.0000 \mathrm{E}+00$ \\
\hline $0.0000 E+00$ & $3.7589 E+\infty 0$ & $0.0000 E+00$ \\
\hline $0.0000 \mathrm{E}+00$ & $0.0000 E+00$ & $2.5000 \mathrm{E}-02]$ \\
\hline$=[3.3$ & $0.0000 E+00$ & $0.0000 E+00$ \\
\hline 0.0000 & $3.0114 \mathrm{E}-01$ & $0.0000 E+00$ \\
\hline $0.0000 \mathrm{E}+00$ & $0.0000 \mathrm{E}+00$ & $7.7316 \mathrm{E}-05]$ \\
\hline
\end{tabular}

Ho Synthesis Control Usage Weighting Factors:

WF - 0.002596 (hour $/ \mathrm{Kg}$ )

AJ $-37.5876\left(1 / \mathrm{M}^{2}\right)$

RCVV -0.25 (1/degrees)

Ho Synthesis Control Rate Weighting Factors:

WF - 7.333E-05 (seconds/(Kg/hour))

AJ -1.96203 (seconds/M²)

RCVV - 0.01176 (seconds/degrees)

$H \infty$ Sensitivity Weighting Functions:

OPR $-(6.7002 S+1000) /(67.002 S+1.0)$

$\mathrm{EPR}-(7.1788 \mathrm{~S}+1000) /(71.788 \mathrm{~S}+1.0)$

$\mathrm{N} 2-(3.5894 \mathrm{~S}+1000) /(35.894 \mathrm{~S}+1.0)$

$H \infty$ Complimentary Sensitivity Weighting Functions:

OPR - (5.5558E-02 S +0.001)/(5.5558E-04 S + 1.0)

$E P R-(5.9527 E-02 S+0.001) /(5.9527 E-04 S+1.0)$

$\mathrm{N} 2-(2.9763 \mathrm{E}-02 \mathrm{~S}+0.001) /(2.9763 \mathrm{E}-04 \mathrm{~S}+1.0)$

Hoo Command Scaling:

$\begin{array}{lll}{\left[\begin{array}{lll}1.6183 & 0.0 & 0.0\end{array}\right]} & 0.0\end{array}$

$\begin{array}{lll}0.0 & 0.2553 & 0.0\end{array}$

$\left.\begin{array}{lll}0.0 & 0.0 & 370.4\end{array}\right]$

$H \infty$ Output Scaling:

$\begin{array}{lll}0.6179 & 0.0 & 0.0\end{array}$

$\begin{array}{lll}0.0 & 3.9170 & 0.0\end{array}$

$\left.\begin{array}{lll}0.0 & 0.0 & 0.0027\end{array}\right]$ 


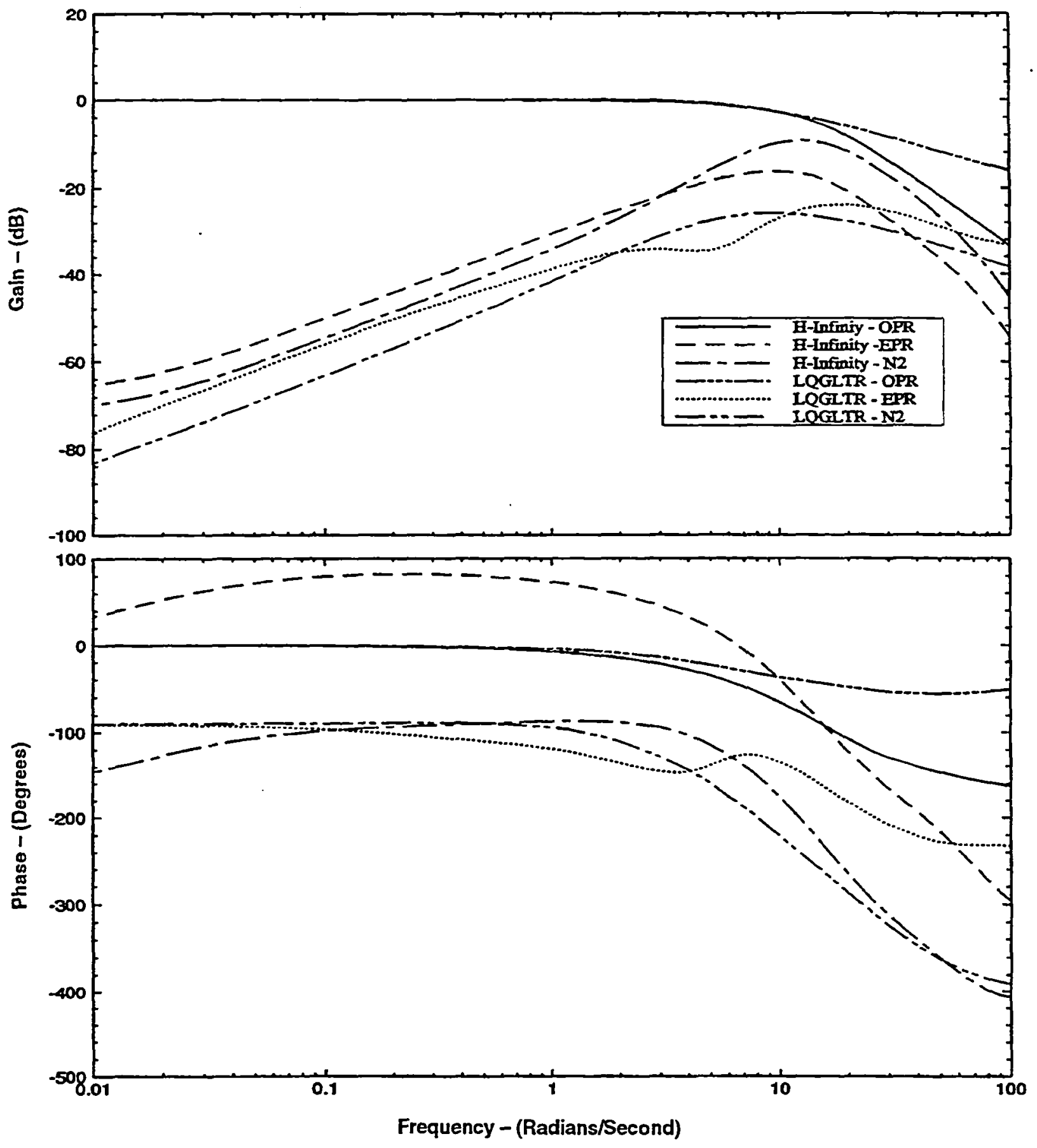

FIGURE 5. CLOSED LOOP FREQUENCY RESPONSE FOR OPR COMMAND 
$\cdot$
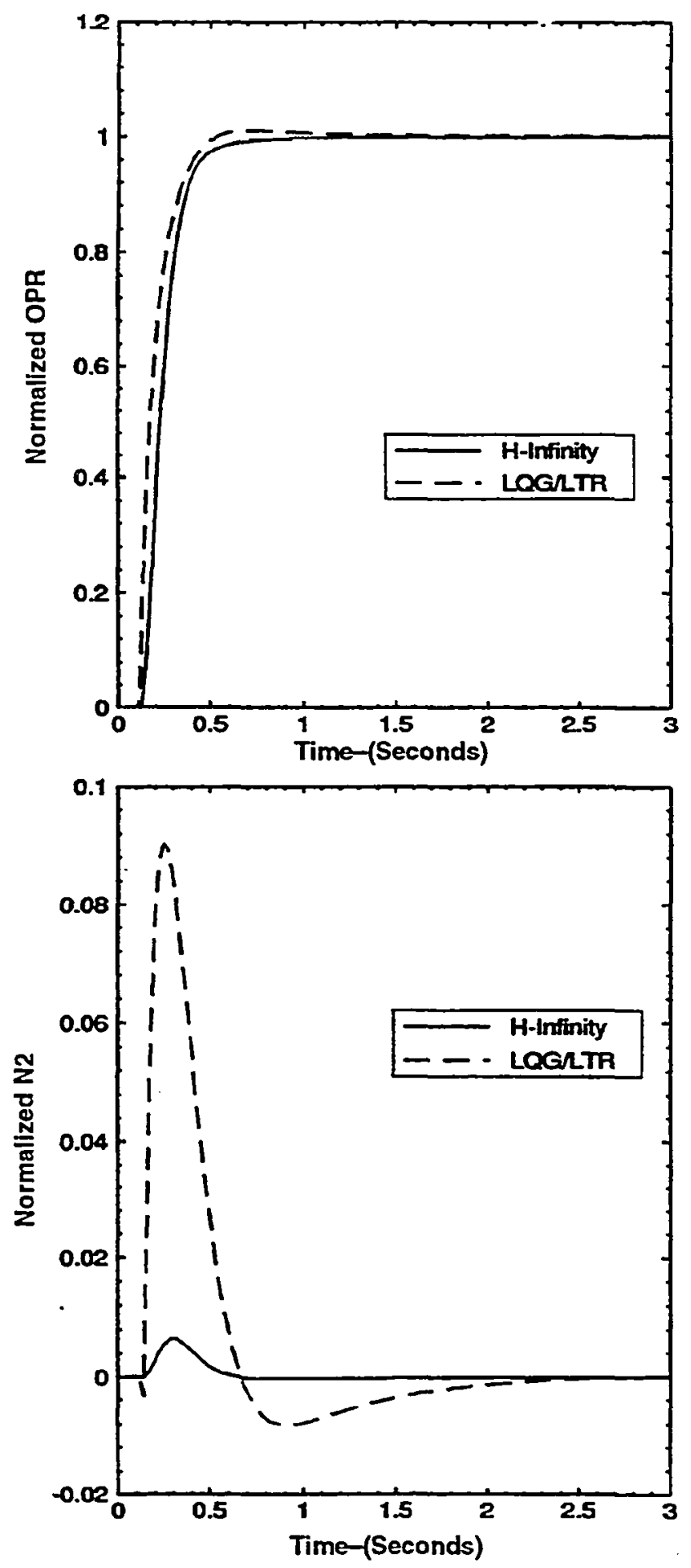

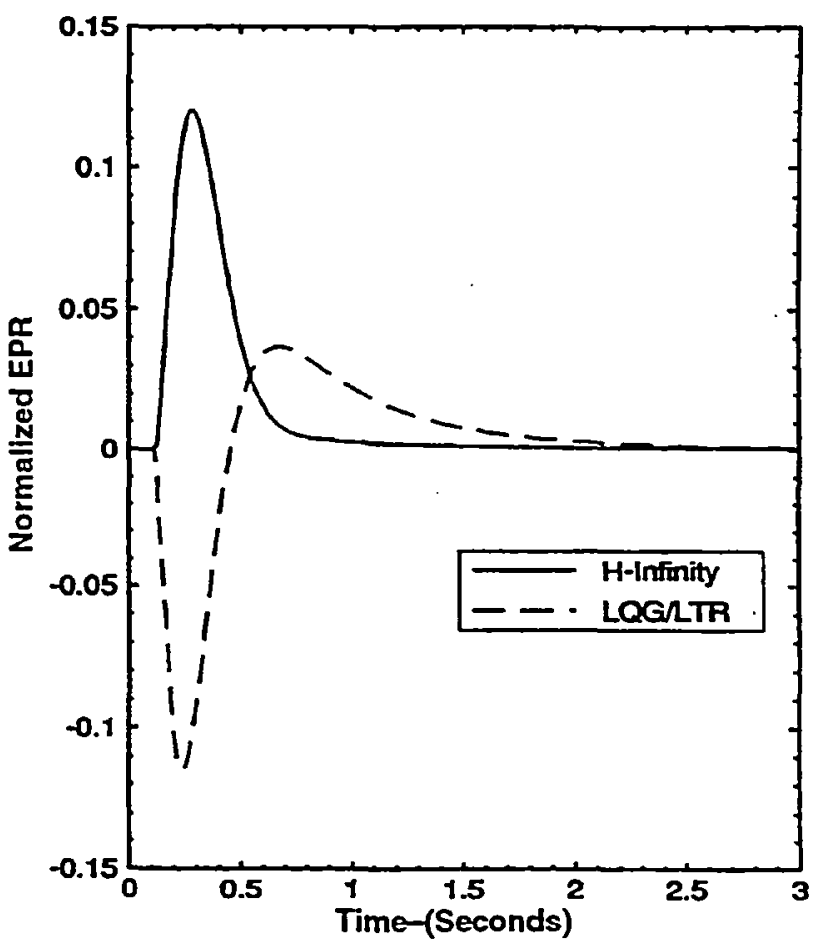

FIGURE 6. CONTROL LOOP RESPONSES TO OPR COMMAND STEP 

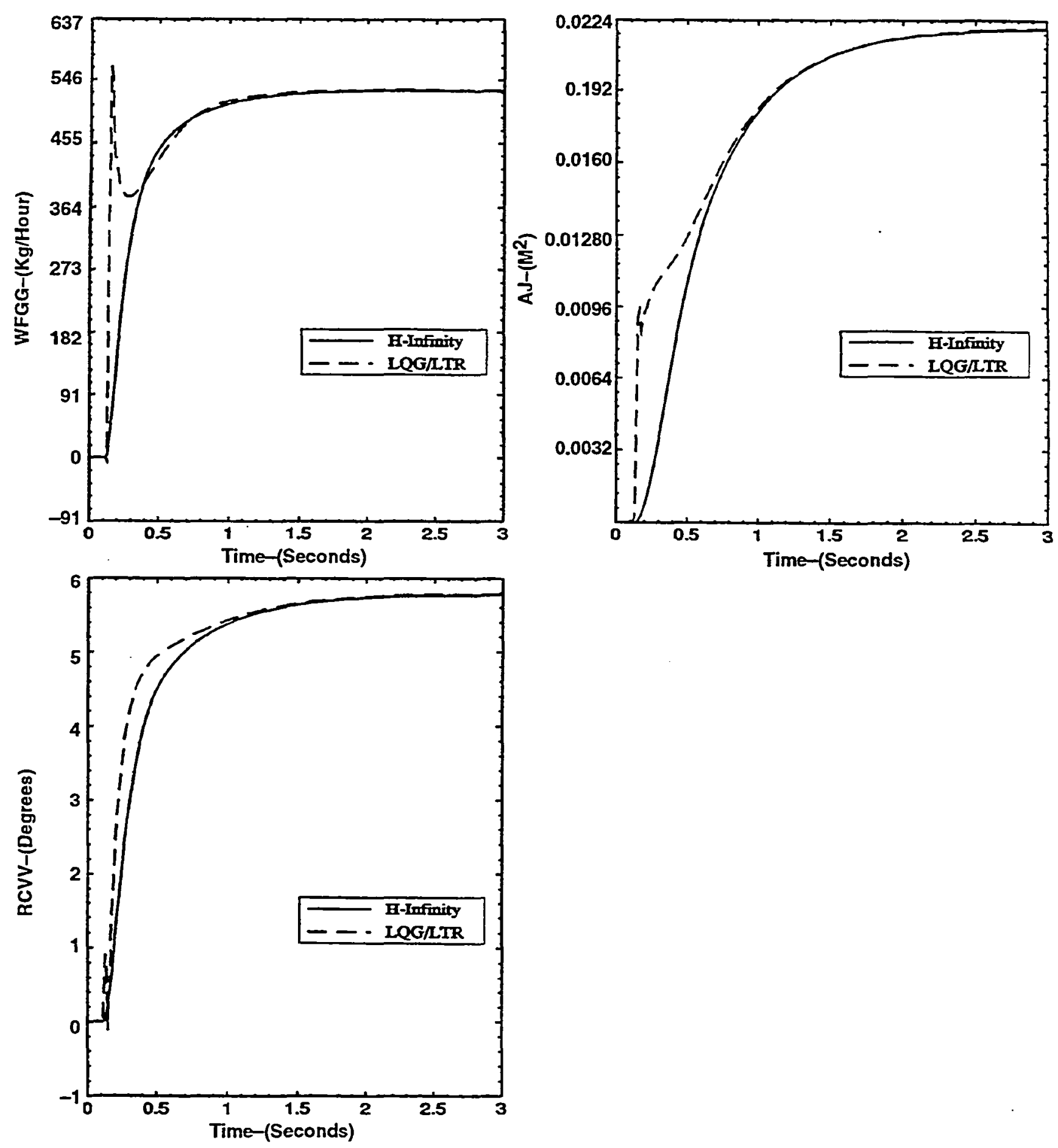

FIGURE 7. CONTROL LOOP OUTPUT RESPONSES TO OPR COMMAND STEP 

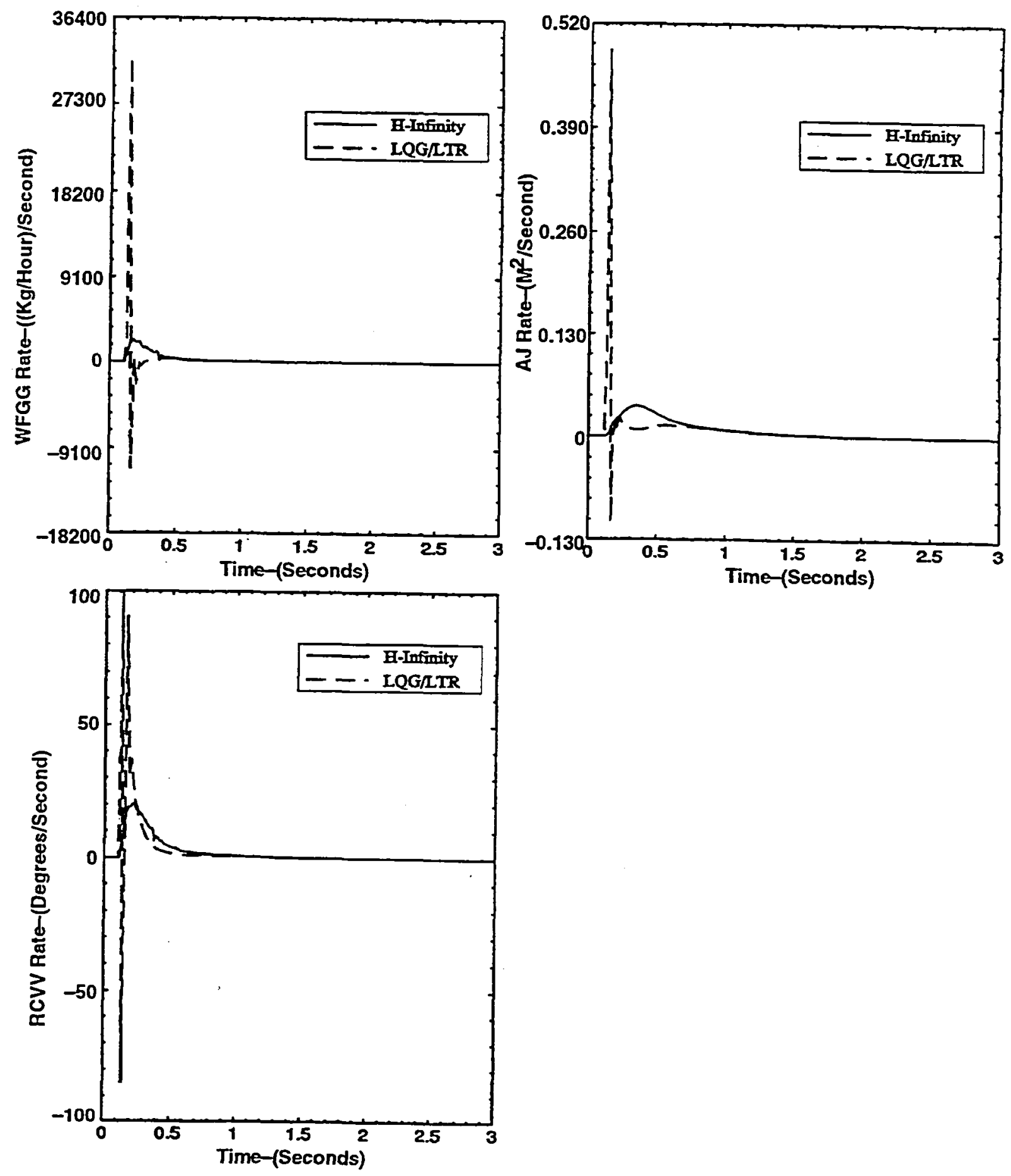

FIGURE 8. CONTROL OUTPUT RATE RESPONSES TO OPR COMMAND STEP 


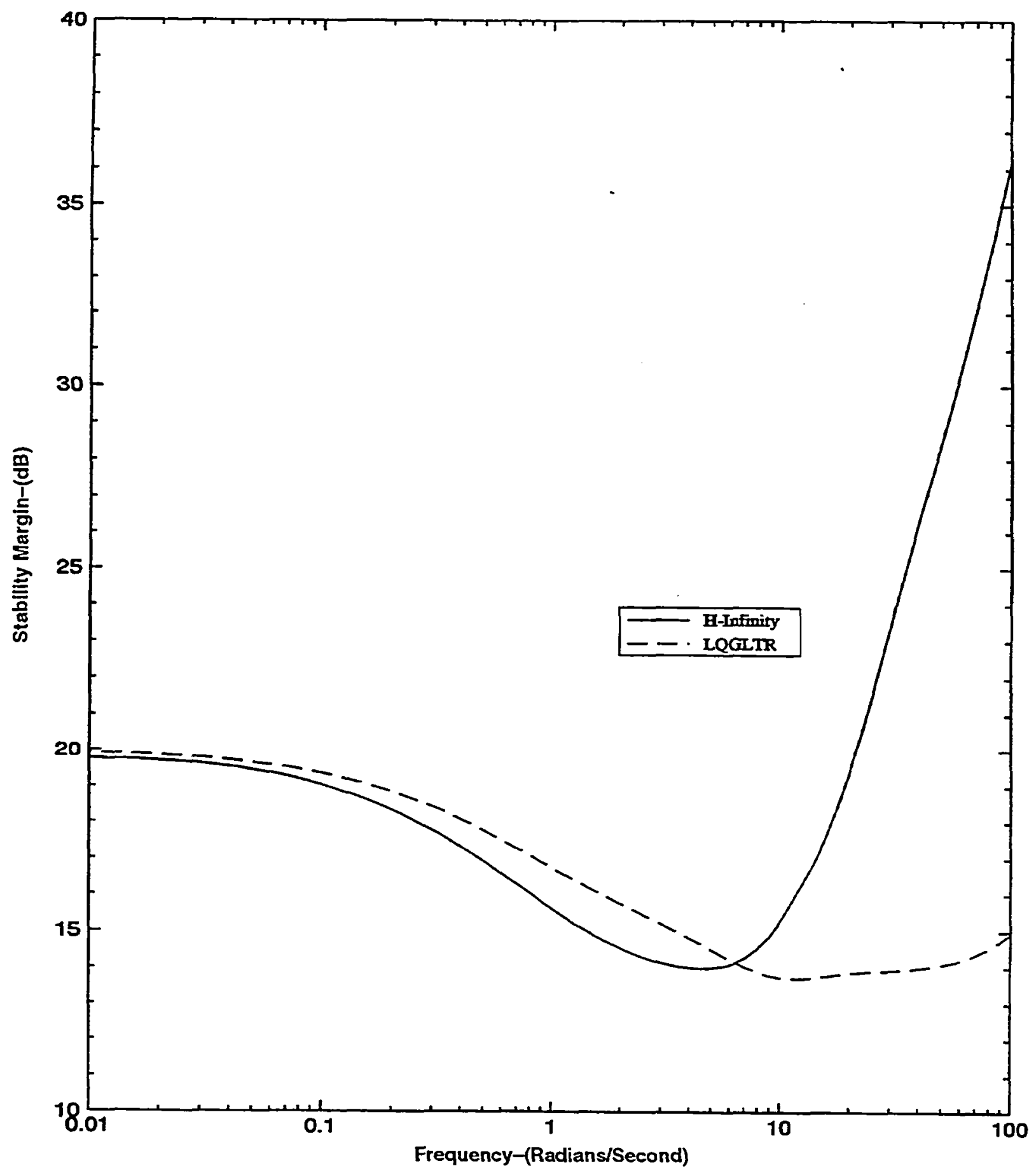

FIGURE 9. STABILITY MARGIN ANALYSIS COMPARISON 
Public reporting burden for this collection of information is estimated to average 1 hour per response, including the time tor reviewing instructions, searching existing data sources, gathering and maintaining the data needed, and comoleting and reviowing the collection of information. Send comments regarding this burden estimate or any Weadquarters Servicos, Directorate for Information Operations and Reports, 1215 Jefterson Davis Highway, Sulte 1204, Arlington, VA 22202-4302, and to the Office of Management and Budget, Papenwork Reduction Project (0704-0188). Washington. DC 20503.

\begin{tabular}{|l|l|l|}
\hline 1. AGENCY USE ONLY (Leave blank) & $\begin{array}{c}\text { 2. REPORT DATE } \\
\text { September } 1995\end{array}$ & $\begin{array}{r}\text { 3. REPORT TYPE AND DATES COVERED } \\
\text { Technical Memorandum }\end{array}$ \\
\hline
\end{tabular}

4. TITLE AND SUBTIILE 5. FUNDING NUMBERS

A Comparison of Multivariable Control Design Techniques for a Turbofan Engine Control

6. AUTHOR(S)

WU-505-62-50

Stephen R. Watts and Sanjay Garg

7. PERFORMING ORGANIZATION NAME(S) AND ADDRESS(ES)

8. PERFormung ORganization REPORT NUMBER

National Aeronautics and Space Administration

Lewis Research Center

Cleveland, Ohio 44135-3191

E-9915

9. SPONSORINGMONITORING AGENCY NAME(S) AND ADDRESS(ES)

10. SPONSORINGMONITORING AGENCY REPORT NUMBER

National Aeronautics and Space Administration

Washington, D.C. 20546-0001

NASA TM-107060

ASME 95-GT-258

\section{SUPPLEMENTARY NOTES}

Prepared for the 40th Gas Turbine and Aeroengine Congress and Exposition sponsored by the American Society of Mechanical Engineers, Houston, Texas, June 5-8, 1995. Stephen R. Watts, United Technologies Pratt \& Whitney, Government Engine and Space Propulsion, West Palm Beach, Florida (work funded under NASA Contract NAS3-26618), and Sanjay Garg, NASA Lewis Research Center. Responsible person, Sanjay Garg, organization code 2550, (210) 433-2355.

12a. DISTRIBUTIONAVAILABILTTY STATEMENT 12b. DISTRIBUTION CODE

Unclassified - Unlimited

Subject Categories 08, 07, and 63

This publication is available from the NASA Center for Aerospace Information, (301) 621-0390.

13. ABSTRACT (Maximum 200 words)

This paper compares two previously published design procedures for two different multivariable control design techniques for application to a linear engine model of a jet engine. The two multivariable control design techniques compared were the Linear Quadratic Gaussian with Loop Transfer Recovery (LQG/LTR) and the H-Infinity $(H \infty)$ synthesis. The two control design techniques were used with specific previously published design procedures to synthesize controls which would provide equivalent closed loop frequency response for the primary control loops while assuring adequate loop decoupling. The resulting controllers were then reduced in order to minimize the programming and data storage requirements for a typical implementation. The reduced order linear controllers designed by each method were combined with the linear model of an advanced turbofan engine and the system performance was evaluated for the continuous linear system. Included in the performance analysis are the resulting frequency and transient responses as well as actuator usage and rate capability for each design method. The controls were also analyzed for robustness with respect to structured uncertainties in the unmodeled system dynamics. The two controls were then compared for performance capability and hardware implementation issues.

14. SUBJECT TERMS

Propulsion control; Multivariable control

17. SECURTIY CLASSIFICATION OF REPORT Unclassified
18. SECURTY CLASSIFICATION OF THIS PAGE Unclassified
19. SECURITY CLASSIFICATION OF ABSTRACT Unclassified 